\title{
THE PAH HYPOTHESIS
}

Spectroscopic properties of large aromatic molecules in the infrared

\author{
L. D'HENDECOURT AND E. DARTOIS \\ Institut d'Astrophysique Spatiale, Bat 121 \\ Université Paris XI, 91405 ORSAY CEDEX, France
}

\begin{abstract}
Polycyclic Aromatic Hydrocarbons (PAHs) are undoubtedly present in large amounts in many different astronomical objects as well as in the diffuse interstellar medium. They are detected through their infrared vibrational transitions in the mid-infared but are expected to be observable in other regions of the spectrum such as the UV, the visible and the far-infrared. PAHs are suggested to be the most abundant free organic molecules in the interstellar environment. In the laboratory, investigations have been undertaken to provide the tools for the interpretation of astronomical observations. For example, the recent detection of the overtone of the well-known $3.28 \mu \mathrm{m}$ band around $1.67 \mu \mathrm{m}$ has led to the determination of a relatively large minimum average size of about 60 atoms. In the far-infrared, above 15 up to $100 \mu \mathrm{m}$, expected transitions from laboratory measurements are scattered all over this region, making the detection of these lines difficult. In the diffuse medium, a complex aromatic network is expected on interstellar grains and its similarity with carbon extracts from primitive meteorites is suggestive of a link between interstellar matter and primitive Solar System bodies, in particular comets.
\end{abstract}

\section{Introduction and background}

PAHs are present in the interstellar medium as a mixture of neutral and ionized species, and responsible for the set of mid-infrared emission bands at $3.28,6.2,7.7,8.6$ and $11.3 \mu \mathrm{m}$ which are observed in many different objects such as reflection and planetary nebulae as well as in external galaxies where, in some cases, they dominate the infrared emission of the entire galaxy, such as in M82. Although neutral PAHs have been first proposed by Léger \& Puget in 1984, ionized ones have also to be considered (Allamandola et al. 1985, Szczepanski \& Vala 1993, Hudgins et al. 1994). 
These mid infrared emission lines are attributed to the vibrational decay of large molecular species following the absorption of single UV photons. The observed bands do indeed correspond to fundamental vibrations of large aromatic molecules such as the $=\mathrm{C}-\mathrm{H}$ stretch at $3.28 \mu \mathrm{m}$. Needless to say that a precise identification with a given molecule is hopeless since vibrational transitions pertain to molecular subunits, so that the observed species reflect the presence of a large family of aromatic molecules (Bellamy 1966). The fact that the transitions occur all at the same wavelengths in the mid-infrared, is the main reason for the detection of these lines in astronomical objects. In the far-infrared, the situation is more complicated as shown in the intensive study by Moutou et al (1996). The presence of PAHs in the diffuse medium is also indicated by the $12 \mu \mathrm{m}$ IRAS Cirrus emission (Boulanger et al. 1985) and by the detection of the 3.28 and 6.2 $\mu \mathrm{m}$ lines in the same clouds later on by Giard et al. (1988) and Ristorcelli et al. (1994). From a knowledge of their absorptions cross-sections in the UV, measured in the gas phase using a UV synchrotron radiation source (Joblin et al. 1992), and a precise estimate of the energy released in the infrared emission bands, the amount of cosmic carbon in large aromatic molecules is estimated to be about $15 \%$, a fairly high number. The reason for the presence of PAHs in the diffuse ISM is probably related to the high stability of these molecules as is well known in photophysics (Birks 1970) and photochemistry (Clar 1964). Finally, an absorption feature pertaining to the aromatic $=\mathrm{C}-\mathrm{H}$ stretch has been observed by Sellgren et al. (1995) towards a protostar, Mon R2/IRS3. From this observation, an abundance of $9 \%$ of cosmic carbon in PAHs was derived. A significant fraction of $\mathrm{PAH}$ molecules can be ionized and partly dehydrogenated in regions where the UV field is intense. Calculations have shown that the degree of ionization is high in planetary and reflection nebulae (d'Hendecourt \& Léger 1987). The situation is however less clear for the diffuse interstellar medium where recent calculations point towards more neutral (Bakes \& Tielens 1994) or even negatively charged species (Dartois \& d'Hendecourt 1997). This last point is important if one wants to reconcile the PAH hypothesis with the suggestion that they may be at the origin of the Diffuse Interstellar Bands. In this paper, we concentrate on some recent observations and laboratory data that confirm more solidly that PAHs are indeed gas phase molecules. We present also some far infrared data on PAHs and finally discuss the connection of interstellar PAHs with solid state organic molecules observed in the diffuse interstellar medium and recovered in primitive carbonaceous meteorites.

\section{Detection of the overtone of the $3.3 \mu \mathrm{m}$ emission feature}




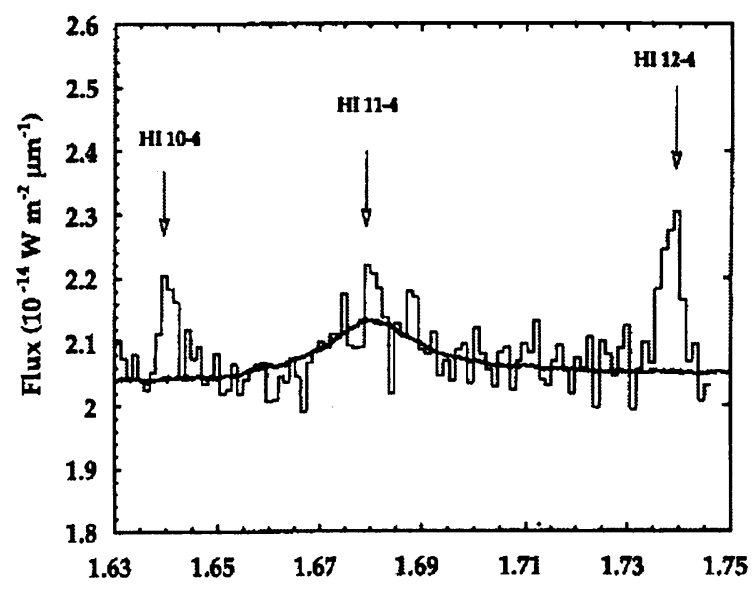

Figure 1. The spectrum of IRAS $21282+5050$ compared to a laboratory absorption spectrum of the $0-2 \mathrm{CH}$ stretch in gas phase pyrene at $800 \mathrm{~K}$ (Geballe et al. 1994). The spectrum also shows $\mathrm{H}$ recombination bands at $1.641,1.681$ and $1.737 \mu \mathrm{m}$.

A key parameter to understand the nature of the emitting aromatic molecules is the emission temperature. Since the model implies that the molecules are heated by the absorption of a single UV photon, the knowledge of the absorbed energy, equated to the specific heat of the emitter allows to derive an estimate of the molecular sizes. This was attempted by Léger et al. (1989), using an emission model and the ratio of two bands pertaining to the same molecular subunit, namely $=\mathrm{C}-\mathrm{H}$ (stretching and bending). A mean size of 50 atoms was then derived. Using a similar approach and ratios of 3.3/11.3 micron bands in 20 IRAS sources, larger size estimates (90/140) were derived by Jourdain de Muizon et al. (1990a). However, since the identification of the $11.3 \mu \mathrm{m}$ band is difficult (the problem of dehydrogenation remains unsolved), it is highly desirable to obtain an estimate of the PAH size which does not rely on this line. In contrast, the $3.28 \mu \mathrm{m}$ line corresponds to the $\mathrm{v}=1 \rightarrow 0$ vibrational transition and, since the molecule is highly excited, following the absorption of the UV photon, it is possible to observe emission from the $\mathrm{v}=2$ level. These two transitions coming from the same vibration mode, their intensity ratio will be governed by the thermal population of the levels. Thus, a size estimate may be derived provided the oscillator strength of the two bands is known. This overtone might be observable in astronomical objects. It is well known that the $=\mathrm{C}-\mathrm{H}$ stretch is highly anharmonic so that this transition is weak but not totally forbidden. Careful gas phase studies of PAHs at elevated 


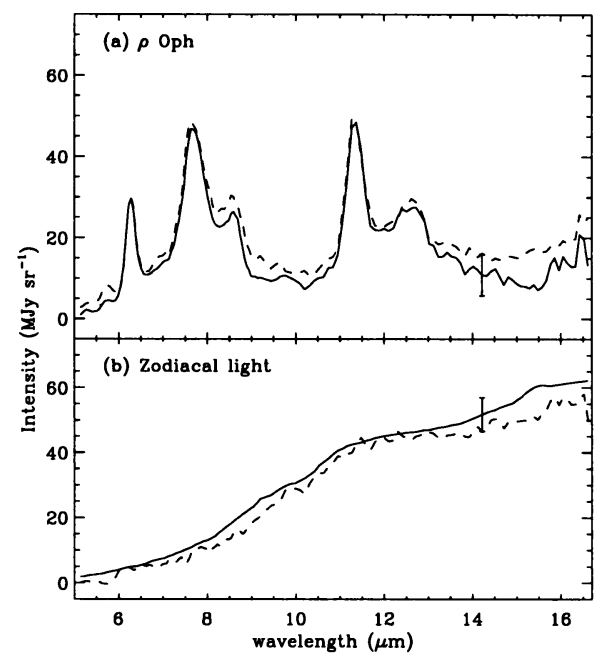

Figure 2. Mean emission spectrum within the $\rho$ Oph field. The solid line is the spectrum derived from data averaging, the dashed line from correlations analysis. The difference between the two spectra corresponds to a $10 \%$ variation in the continuum associated with the zodiacal light substraction (Boulanger et al. 1996).

temperatures have been undertaken by Joblin et al. (1995) who have been able to measure the strength of the 2 - 0 transition in absorption. Geballe et al. (1994) have successfully found the expected transition in the bright object IRAS $21282+5050$ at $1.67 \mu \mathrm{m}$ as reproduced in Figure 1. A mean size of 60 atoms is found for PAHs in this object. Finally, the presence of the overtone band allows to estimate the contribution of the so-called "hot band", the 2 - 1 transition in the $3.4 \mu \mathrm{m}$ region. This region contains the hot-band but also transitions expected from molecular subgroups such as -CH2 and -CH3 (Jourdain de Muizon et al. 1990b) as well as a possible contribution from the aliphatic-C-H stretch arising in super-hydrogenated PAHs as recently proposed by Bernstein \& Allamandola (1996).

\section{Recent ISO observations}

ISO observations using ISOCAM have measured the dust emission at the northern part of the dense molecular cloud Ophiuchus, where the exciting UV radiation field is estimated to be only 10 times the mean solar neighborhood value. The IR spectrum shows a clear signature of the PAH bands (Boulanger et al. 1996). The spectrum between 5 and $16 \mu \mathrm{m}$ is displayed in Figure 2 and shows a continuum below the individual PAH features between 8.6 and $11.3 \mu \mathrm{m}$ and beyond the $12.7 \mu \mathrm{m}$ band. The intensity of the continuum is strongly correlated with the emission bands, which is 


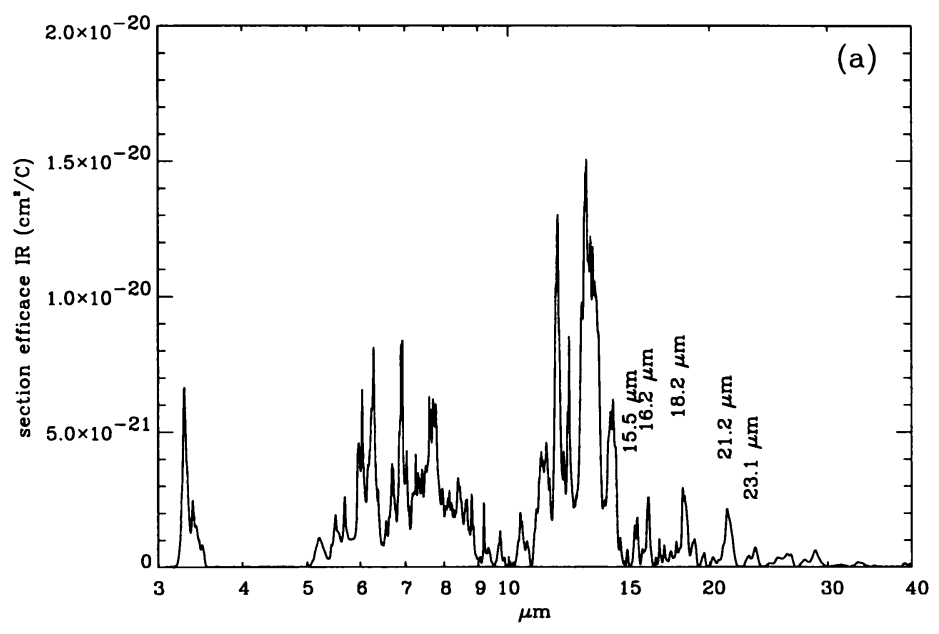

Figure 3. NIR and FIR absorption spectrum composed of a 40 PAHs mixture. The accumulation in the peak positions is displayed (Moutou et al. 1996).

in support of a physical link between the emitters of the continuum and those of the bands. The ISOCAM observations in Ophiuchus, a region of low UV radiation field, displaying clearly the UIR bands are in strong support of the PAH hypothesis since only molecular size emitters can account for such an out of equilibrium process. The temperature of larger grains would indeed be too low to allow emission at $3.28 \mu \mathrm{m}$.

\section{PAHs in the far-infrared}

In contrast to mid-infrared absorption (or emission) features, far infrared properties from 15 to $100 \mu \mathrm{m}$ are poorly known. Since up to now spectral information from the interstellar medium in this wavelength range was scarce, the poor amount of data on PAHs was not considered as a problem. In preparation for ISO observations which may change the situation quite drastically, the FIR spectra of about 40 different large molecular PAH species have been undertaken in our laboratory. The results do show some possibly detectable features as evidenced in Moutou et al. (1996). Basically, the far-infrared range may be divided in two regions. From 15 to $40 \mu \mathrm{m}$, although absorption bands are scattered through the whole range, accumulation peaks are observed. Four modes are indeed prominent, they occur at 16.2, 18.2, 21.2 and $23.1 \mu \mathrm{m}$. This result is shown in Figure 3 where a composite absorption spectrum of $40 \mathrm{PAHs}$ is presented for the far-IR $(14-40 \mu \mathrm{m})$ while the $3-4 \mu \mathrm{m}$ region implies only compact PAHs. 
Over $40 \mu \mathrm{m}$, up to $100 \mu \mathrm{m}$, no accumulation peaks are observed and the $\mathrm{PAH}$ absorptions amount to some sort of weak continuum, valid for any mixture. Clearly from this work, new emission features might be expected from ISO data but one will easily note that this approach may not allow a precise molecular identification. Going to the far-IR, skeleton modes of PAHs (analogous to lattice vibrations in a solid) are expected to be responsible for these IR activity (see Cyvin 1982 and references therein). These modes do depend on the particular molecular geometry and will be highly sensitive on the precise nature of the molecule (presence of hetero atoms, dehydrogenation...). Consequently, observations of possible broad emission bands with ISO may be difficult to relate to any molecular species, a situation that resembles the one encountered for the Diffuse Band problem where electronic transitions of large molecules are extremely sensitive to the molecular orbitals geometry which renders any identification extremely difficult.

\section{Theoretical considerations}

Destruction of PAHs probably involves double ionization of PAHs (Leach 1987). Jochims et al. (1993), argue that nascent PAHs ions of any size formed by photoionization will preferentially relax by IR emission. Those PAHs that have less than 30 atoms tend to relax by photofragmentation when ionized twice. The double ionization potential of 28 PAHs has recently been measured by Tobita et al. (1994). These data show that small PAHs have in general an ionization potential from cation to dication above $12 \mathrm{eV}$ and the majority above $13 \mathrm{eV}$. Larger molecules have in general a smaller $\Delta \mathrm{I}$ but can distribute the energy efficiently over the larger surface area and are therefore more stable. Allain et al (1996 a,b) have studied the photo-stability of PAHs in regions of high UV radiation fields where IR emission bands are observed (such as reflection and planetary nebulae, HII regions). Their results show that only PAHs with a size in excess of 50 carbon atoms may survive the UV radiation field in these regions, a size which is in remarkable agreement with the one derived from the detection of the overtone of the 3.28 micron band by Geballe et al. (1994). PAHs such as coronene and ovalene, although known to be very stable molecules (Clar 1964) should be destroyed even in the diffuse interstellar medium, according to Allain et al. (1996 a, b). This interesting result may explain why attempts to detect coronene and ovalene ions, widely studied in neon matrices (Ehrenfreund et al. 1992), in the diffuse medium has failed (Ehrenfreund et al. 1995). All in all, if PAHs are large (60 atoms) it is quite striking to note that these species have yet to be spectroscopically studied in the laboratory, particularly in the visible as an attempt to solve the problem 


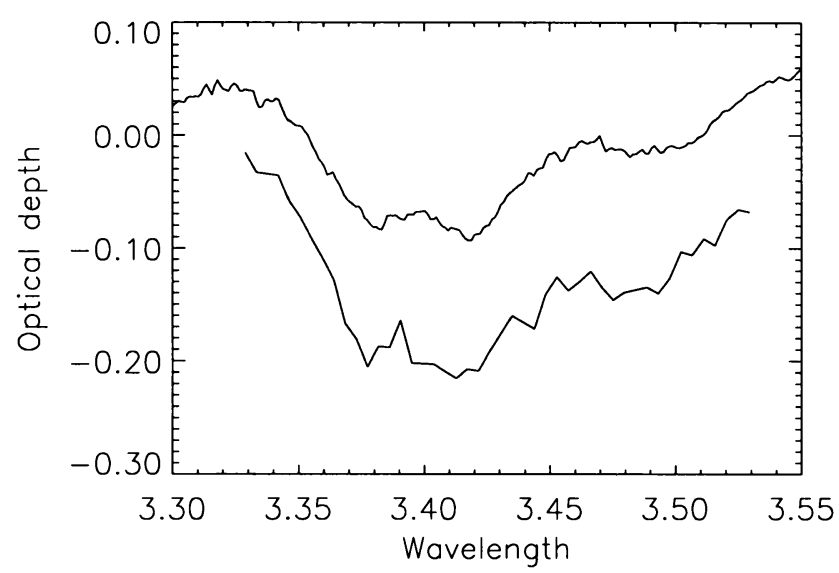

Figure 4. The IR spectrum of the $3.4 \mu \mathrm{m}$ features of an organic residue of the Orgueil meteorite (upper curve) in comparison with the interstellar high resolution measurements towards IRS 6E (taken from Pendleton et al. 1991). The upper spectrum have been shifted for further clarity. Note that $1 \mathrm{mg}$ of the carbon extract from Orgueil over a 13 $\mathrm{mm} \mathrm{KBr}$ pellet was necessary to reproduce the optical depth observed towards IRS6 E. The asymmetric and symmetric stretching modes of the -CH3- groups appear at 3.38 and $3.48 \mu \mathrm{m}$; the -CH2-group (in alkanes) at 3.42 and $3.50 \mu \mathrm{m}$, respectively. The two bands at 3.42 and $3.50 \mu \mathrm{m}$ are blended into one band. This fit suggests a similar chemical composition or structure of the interstellar organic matter and that of the meteoritic polymer.

of the Diffuse Bands.

\section{Solid state complex organic matter in the interstellar medium}

PAHs have been identified in meteorites, interplanetary dust particles and in comets (Hahn et al. 1988, Clemett et al. 1993, Encrenaz et al. 1988). The infrared absorption spectra of semi-anthracite coal grains reproduce well the $2-13 \mu \mathrm{m}$ emission spectra of carbon-rich post AGB stars (Guillois et al. 1996). The spectral changes in the evolution of coals are also found in carbonaceous chondrites. The spectrum of IRS 7, a late supergiant located close to the Galactic Center presents the signature of aliphatic organic structures on grains around $3.4 \mu \mathrm{m}$ (Sandford et al. 1991). The 3.4 $\mu \mathrm{m}$ absorption was also detected in a circumstellar envelope (Lequeux \& Jourdain de Muizon 1990) and Wolf-Rayet stars (Sandford et al. 1991). The detection of the $3.4 \mu \mathrm{m}$ absorption feature towards NGC1068, which has an active nucleus obscured by dust and the detection of this feature towards a Seyfert galaxy, 08572+3915, offers exciting perspectives (Bridger et al. 1996). The strong consistency in spectral signatures in different galaxies shows that common carbonaceous structures are formed. The 3 micron absorption spectrum of the Galactic Center source IRS7 has been compared 
to that obtained from a deuterium rich organic extract from the Orgueil carbonaceous meteorite (Ehrenfreund et al. 1991). The meteoritic material consists of a kerogen structure, dominated by an aromatic backbone. The very close match between the two spectra in the $3.4 \mu \mathrm{m}$ region suggests that the chemical composition of the interstellar grains closely resembles that of the meteoritic macromolecule. Assuming that the $\mathrm{C} / \mathrm{H}$ ratio in this meteoritic material is the same as in the ISM "kerogen", it was calculated that up to $50 \%$ of the cosmic carbon could be depleted in the diffuse ISM in the form of this kerogen-like material (Ehrenfreund et al. 1991). Figure 4 shows a comparison between the spectrum of the insoluble organis residue from Orgueil with new high resolution data of another Galactic Center source, IRS6 E, obtained by Pendleton et al. (1994). Note that the meteorite carbon extract has a $\mathrm{D} / \mathrm{H}$ ratio largely enriched in deuterium, which suggests that this material has been incorporated directly from the interstellar medium. Naturally, due to obvious observational constraints (the $5-8 \mu \mathrm{m}$ region is unaccessible from the ground), one should notice that the real "fingerprints" of the molecular composition of the material to IRS7 and IRS6, has not yet been available for analysis. Once again, much is expected from ISO, in particular to understand if this material is aliphatic or aromatic in nature, this last composition being the one recorded in the meteorite. Preliminary results on IRS7 show clearly that the information contained in ISO-SWS spectra is very rich but, up to now, not fully exploited (Lutz et al. 1996).

\section{Conclusion}

The ubiquitous presence of the signature of aromatic molecules has been confirmed with preliminary ISO measurements. Mid-infrared imaging spectroscopy with ISOCAM shows that out-of-equilibrium infrared emission in the "classical" Unidentified Infrared Bands is easily detected in regions of low UV radiation density, where large grains are unable to emit, thus pointing to the presence of gas phase free flyers molecules. A major difficulty remains the precise identification of given molecular species (e.g; coronene or ovalene). The mid-infrared region of the spectrum reveals a family of molecules without the hope to identify any particular species (unless one is strongly dominating). We have shown that far-infrared transitions might be detectable by ISO under $40 \mu \mathrm{m}$ but a precise identification of molecular species in the far-infrared remains a difficult task, as challenging as the problem of the Diffuse Interstellar Bands in the visible. The detection of the overtone of the $3.28 \mu \mathrm{m}$ band points to rather large molecules (60 atoms and over), at least in one object. Such a result is consistent with theoretical and experimental studies about the photostability of PAHs. It also points 
to rather crucial problems for laboratory experiments since up to now, smaller molecules have been studied. Finally, there appears to be a strong link between the organic material observed in the diffuse ISM and in carbon extracts from primitive meteorites. This last point may be spectroscopically elucidated when more ISO-SWS data will allow a strict comparison of both spectra over an as large as possible wavelength domain. From this kind of approach as well as from isotopic measurements performed "in-situ" in various meteoritic samples, it may be said that interstellar matter is already available in terrestrial laboratories.

\section{Acknowledgements}

We are grateful to F. Boulanger for providing us with the recent ISOCAM spectra of $\rho \mathrm{Oph}$ and to Y. Pendleton for the high resolution spectrum of IRS6 E. We thank P. Ehrenfreund for a critical reading of the manuscript and C. Moutou for providing the data on the far IR properties of PAHs.

\section{References}

Allain T., Leach S., Sedlmayr E., 1996a, A \& A 305, 602.

Allain T., Leach S., Sedlmayr E., 1996b, A \& A 305, 616.

Allamandola L.J., Tielens A.G.G.M., Barker J.R., 1985, ApJ 290, L25.

Bakes E. L. O., Tielens, A.G.G.M. 1994, ApJ 427, 822.

Bellamy L.J., 1966, IR spectra of complex molecules, Wiley New York.

Bernstein M. , Allamandola L.J., 1996, ApJ, in press.

Birks J.B., 1970, Photophysics of aromatic molecules, Wiley Interscience, London.

Boulanger F., Baud B., van Albada G.D. 1985, A\&A 144, L9.

Boulanger, F. et al., 1996, A \& A 315, L325.

Bridger A., Wright G., Geballe T., 1993, in: IR Astronomy with Arrays. The next generation.

Clar E., 1964, Polycyclic Hydrocarbons, Academic Press London.

Clemett S.J., Maechling C.R., Zare R.N., Swan P.D., Walker R.M., 1993, Science 262, 721.

Cyvin S.J., 1982, J. of Mol. Struct., 79, 423.

Dartois E., d'Hendecourt L. 1997, A \& A, in press.

Ehrenfreund P., Robert F., d'Hendecourt L., Behar F., 1991, A \& A, 252, 712.

Ehrenfreund P., d'Hendecourt L., Verstraete L., Léger A., Schmidt W., Defourneau D., 1992, A \& A 295, 257.

Ehrenfreund P., Foing B.H., d'Hendecourt L., Jenniskens P., Desert X., 1995, A \& A, 299, 213.

Encrenaz, Th., d'Hendecourt, L., Puget, J.L., 1988, A \& A 207, 162.

Geballe T.R., Joblin C., d'Hendecourt, L., Jourdain de Muizon, M., Tielens, A.G.G.M., Léger A., 1994, ApJ 434, L 15.

Giard, M., Pajot, F., Lamarre, J.M., Serra, G., Caux, E., Gispert, R., Léger, A., Rouan, D., 1988, A \& A 201, L1.

Guillois O., Nenner I., Papoular R., Reynaud C., 1996, ApJ 464, 810.

Hahn J.H., Zenobi R., Bada J.F., Zare R.N., 1988, Science 239, 1523.

d'Hendecourt L., Léger A., 1987, A \& A 180, L9.

Hudgins D.M., Allamandola L.J., 1995, J. of Phys. Chem. 99, 3033.

Joblin C., Léger A., Martin P., 1992, ApJ 393, L79. 
Joblin C., Boissel P., Léger A., d'Hendecourt L., Défourneau D., 1995, A \& A 299, 835.

Jochims H.W., Ruehl E., Baumgaertel H., Tobita S., Leach S., 1994, ApJ 420, 307.

Jourdain de Muizon M., d'Hendecourt L.B., Geballe T.R., 1990a, A \& A 227, 526.

Jourdain de Muizon M., d'Hendecourt L.B., Geballe T.R., 1990b, A \& A 235, 367.

Leach S. 1987, in Polycyclic Aromatic Hydrocarbons and Astrophysics, eds. Léger A. and d'Hendecourt L., Reidel, Dordrecht, 99.

Léger A., Puget, J.L., 1984, A \& A Lett. 137, L5.

Léger A., d'Hendecourt L., Defourneau D., 1989, A \& A 216, 148.

Lequeux J., Jourdain de Muizon M., 1990, A \& A 240, L 19.

Lutz D. et al. 1996, A \& A 315, L269.

Moutou C., Léger A., d'Hendecourt L., 1996, A \& A 310, 297.

PendletonY, Sandford S.A., Allamandola L.J., Tielens A.G.G.M., Sellgren K., 1994, ApJ $437,683$.

Ristorcelli, I. et al., 1994, A \& A 286, L 23.

Sandford, S.A., Allamandola, L.J., Tielens, A.G.G.M., Sellgren, K., Tapia, M., Pendleton, Y., 1991, ApJ 371, 607.

Sellgren K., Brooke T.Y., Smith R.G., Geballe T.R., 1995, ApJ 449, L 69.

Szczepanski J., Vala, M., 1993, ApJ 414, 646.

Tobita S., Leach S., Jochims H., Ruehl E., Illenberger E., Baumgaertel H., 1994, Canadian Journal of Physics 72, 1060.

\section{Discussion}

Williams: I think the PAH hypothesis is an hypothesis, but the evidence in support is very strong. However, this evidence is associated with regions of high excitation. It is often said that PAHs are widespread in the ISM. What is the evidence for this?

d'Hendecourt: The PAH hypothesis relies on two basic facts, the presence of a set of infrared emission lines which coincide with vibrational transitions of aromatic species and a thorough description of the emission mechanism which implies transient heating of very small grains (or large molecules). The emission lines are observed in extremely diverse and numerous objects, e.g. reflection and planetary nebulae as well as in external galaxies, thus showing that aromatic species are ubiquitous. Observations of infrared cirrus at $12 \mu \mathrm{m}$ by IRAS has shown that very small grains (transiently heated particles) are common in the diffuse medium. Balloon-borne experiments (see text) have given precious spectroscopic information about these cirrus clouds : the 3.28 and $6.2 \mu \mathrm{m}$ lines are detected and have been interpreted by emission from very small aromatic species e.g., PAHs. Finally, recent observations of the aromatic emission lines in low UV density regions by ISOCAM do confirm the presence of PAH molecules, in high abundances in the general interstellar medium. 